\title{
Heterologous expression of pikromycin biosynthetic gene cluster using Streptomyces artificial chromosome system
}

\author{
Hye-rim Pyeon, Hee-Ju Nah, Seung-Hoon Kang, Si-Sun Choi and Eung-Soo Kim*
}

\begin{abstract}
Background: Heterologous expression of biosynthetic gene clusters of natural microbial products has become an essential strategy for titer improvement and pathway engineering of various potentially-valuable natural products. A Streptomyces artificial chromosomal conjugation vector, pSBAC, was previously successfully applied for precise cloning and tandem integration of a large polyketide tautomycetin (TMC) biosynthetic gene cluster (Nah et al. in Microb Cell Fact 14(1):1, 2015), implying that this strategy could be employed to develop a custom overexpression scheme of natural product pathway clusters present in actinomycetes.
\end{abstract}

Results: To validate the pSBAC system as a generally-applicable heterologous overexpression system for a largesized polyketide biosynthetic gene cluster in Streptomyces, another model polyketide compound, the pikromycin biosynthetic gene cluster, was preciously cloned and heterologously expressed using the pSBAC system. A unique Hindlll restriction site was precisely inserted at one of the border regions of the pikromycin biosynthetic gene cluster within the chromosome of Streptomyces venezuelae, followed by site-specific recombination of pSBAC into the flanking region of the pikromycin gene cluster. Unlike the previous cloning process, one Hindlll site integration step was skipped through pSBAC modification. pPik001, a pSBAC containing the pikromycin biosynthetic gene cluster, was directly introduced into two heterologous hosts, Streptomyces lividans and Streptomyces coelicolor, resulting in the production of 10-deoxymethynolide, a major pikromycin derivative. When two entire pikromycin biosynthetic gene clusters were tandemly introduced into the S. lividans chromosome, overproduction of 10-deoxymethynolide and the presence of pikromycin, which was previously not detected, were both confirmed. Moreover, comparative qRT-PCR results confirmed that the transcription of pikromycin biosynthetic genes was significantly upregulated in S. lividans containing tandem clusters of pikromycin biosynthetic gene clusters.

Conclusions: The $60 \mathrm{~kb}$ pikromycin biosynthetic gene cluster was isolated in a single integration pSBAC vector. Introduction of the pikromycin biosynthetic gene cluster into the pikromycin non-producing strains resulted in higher pikromycin production. The utility of the PSBAC system as a precise cloning tool for large-sized biosynthetic gene clusters was verified through heterologous expression of the pikromycin biosynthetic gene cluster. Moreover, this pSBAC-driven heterologous expression strategy was confirmed to be an ideal approach for production of low and inconsistent natural products such as pikromycin in S. venezuelae, implying that this strategy could be employed for development of a custom overexpression scheme of natural product biosynthetic gene clusters in actinomycetes.

Keywords: Streptomyces artificial chromosome, Pikromycin biosynthetic gene cluster, Heterologous expression

*Correspondence: eungsoo@inha.ac.kr

Department of Biological Engineering, Inha University, Incheon 402-751,

South Korea 


\section{Background}

Microbial natural products including secondary metabolites produced by actinomycetes have been a major resource for new drug discovery and development because of their superior structural diversity and complexity [1]. Although identification of entire biosynthetic gene clusters has become relatively straightforward because of genome mining and next generation sequencing, some of the biosynthetic genes are derived from nonculturable organisms or from microorganisms that are not amenable to genetic manipulation and are therefore not easily expressed for target compound identification [2]. To overcome such intrinsic limitations and achieve functional expression of uncharacterized potentiallyvaluable natural product biosynthetic pathways, relatively well-characterized heterologous host expression strategies have been pursued.

The cosmid/fosmid library system has been used extensively for several decades to enable heterologous expression of natural product biosynthetic gene clusters of actinomycetes [3-8]. Recently, several sophisticated heterologous expression approaches have been introduced, including the linear plus linear homologous recombination (LLHR) system [9], transformation-associated recombination (TAR) system [10] and Streptomyces bacterial artificial chromosome (pSBAC) system [11]. Specifically, Yamanaka et al. designed the TAR cloning vector, pCAP01, which consists of a yeast element, an Escherichia coli element, and an actinobacterial element. The marinopyrrole biosynthetic gene cluster $(30 \mathrm{~kb})$ and taromycin A biosynthetic gene cluster $(67 \mathrm{~kb})$ were captured by a TAR system using yeast recombination activity, then functionally expressed in Streptomyces coelicolor [10]. Although a TAR system might be suitable for cloning and expression of a large-sized cryptic gene cluster screened from actinomycetes genome mining, TAR cloning must be conducted in yeast before intergeneric conjugation into Streptomyces. Additionally, Yin et al. [12] cloned a large-sized salinomycin biosynthetic gene cluster by linear plus linear homologous recombination (LLHR) using the Red/ET system and successfully expressed it in $S$. coelicolor. However, because the efficiency of this system is low for a large cluster $(>60 \mathrm{~kb})$, the cluster should be divided for serial assembly. In addition, both TAR and LLHR systems were mediated by homologous recombination, so undesired recombination could appear.

We previously successfully demonstrated that the pSBAC system is as an efficient heterologous expression system with site-specific restriction site insertion, recombinant pSBAC plasmid rescue, and E. coli-Streptomyces intergeneric conjugation. Using this pSBAC system, we isolated a large-sized TMC biosynthetic gene cluster $(80 \mathrm{~kb})$ from Streptomyces sp. CK4412 and expressed it in both homologous and heterologous hosts [13]. Since the pSBAC cloning approach uses the plasmid rescue method, undesired recombination does not appear and overexpression of the target gene cluster is possible through simple antibiotic marker substitution.

In this study, another large-sized polyketide pikromycin biosynthetic gene cluster, which was discovered in early 1950s and examined by many researchers for identification of the mechanism of polyketide elongation and their structural changes [14-16], was cloned and directly expressed in two different heterologous hosts using the pSBAC system. Moreover, tandem integration of the pikromycin cluster-containing pSBAC in Streptomyces lividans resulted in significantly enhanced productivities of both 10-deoxymethynolide and pikromycin, implying that this pSBAC system might be an efficient strategy for functional overexpression of the entire biosynthetic gene cluster of any potentially-valuable low-titer metabolite in actinomycetes.

\section{Results \\ Isolation of pikromycin biosynthetic gene cluster using the pSBAC system}

There were only two examples of pSBAC-driven heterologous expression systems in Streptomyces, meridamycin $(95 \mathrm{~kb})$ and TMC $(80 \mathrm{~kb})$ biosynthetic gene clusters $[11,13]$. While the entire meridamycin gene cluster was captured in a single pSBAC clone via straightforward restriction enzyme digestion because of the presence of unique restriction enzyme $M f e I$ sites in border regions of the meridamycin biosynthetic gene cluster, unique $X b a \mathrm{I}$ restriction sites were precisely inserted at both border regions of the TMC biosynthetic gene cluster within the chromosome of Streptomyces sp. CK4412. Since the pikromycin biosynthetic gene cluster does not possess a unique restriction enzyme site in the borders like the TMC gene cluster, a unique restriction enzyme site was first inserted at the borders of the pikromycin gene cluster on the S. venezuelae ATCC 15439 chromosome. At one side of the pikromycin biosynthetic gene cluster near pikRII, a HindIII restriction enzyme site was inserted into $150 \mathrm{bp}$ upstream region from pikRII using PCR targeted gene insertion [17]. To accomplish this, pMSCpik311 containing an apramycin resistance gene, oriT and a Hin$d \mathrm{III}$ restriction enzyme site was constructed in $E$. coli based on pSCpik311, the pikromycin border-containing cosmid. The modified cosmid was introduced into $S$. venezuelae ATCC 15439 by conjugation, followed by target sequence-specific recombination at the border of the pikromycin gene cluster (Fig. 1). The resulting ex-conjugants were selected with the antibiotic selection marker, and insertion of HindIII in the correct site was confirmed by PCR analysis and sequencing (Additional file 1: Figure 


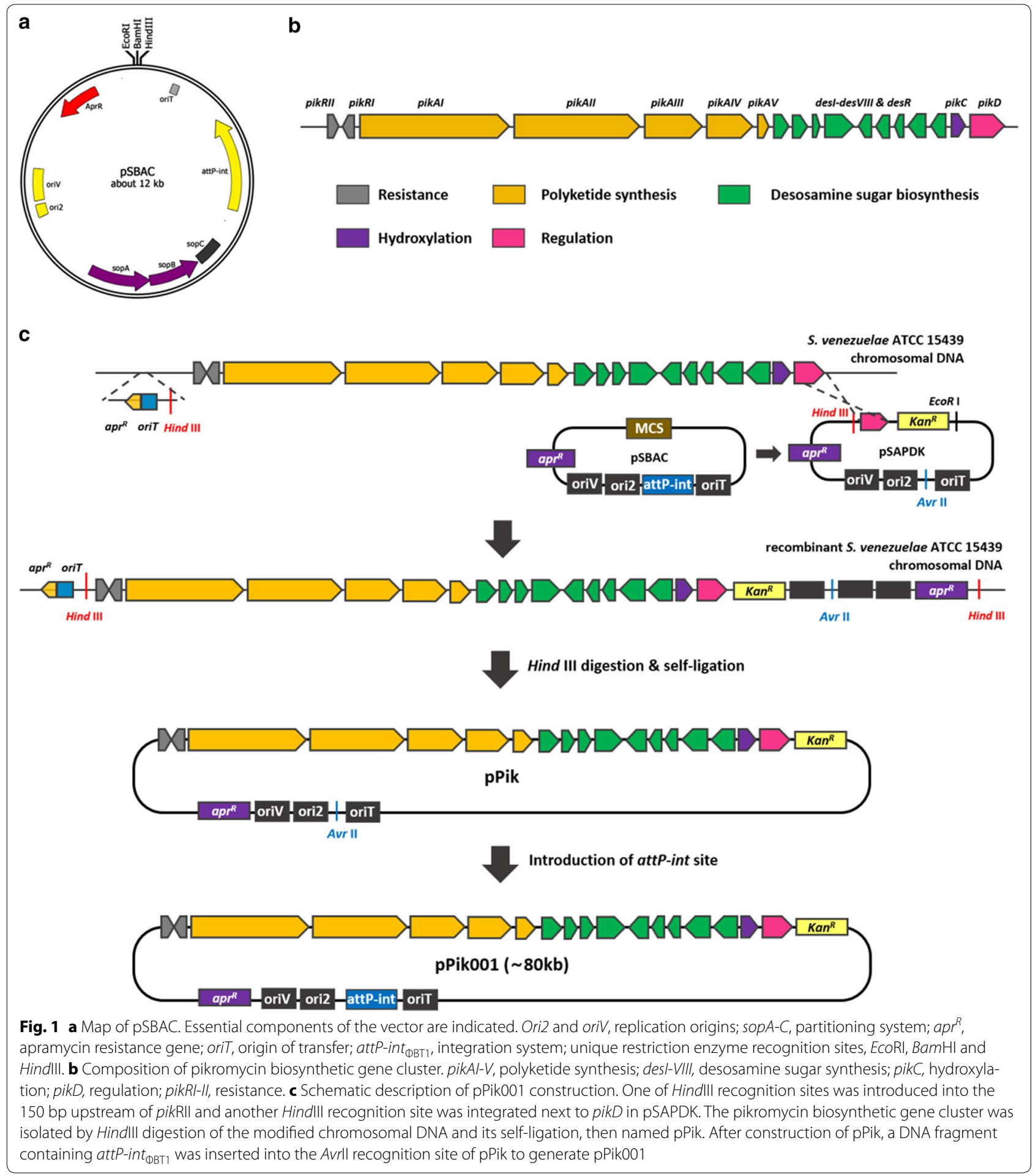

S1). For the other side of the cluster, a HindIII restriction enzyme site in the multiple cloning site of pSBAC was used as a restriction enzyme site for plasmid rescue method in the vicinity of pikD. To integrate a pSBAC vector into the recombinant S. venezuelae ATCC 15439 chromosome, a gene cassette containing a segment of pikD and the kanamycin resistance gene was cloned into a pSA (pSBAC $\triangle a t t P-i n t_{\Phi B T 1}$ ) using the HindIII and EcoRI restriction enzyme sites. The resulting construct was named pSAPDK, then directly introduced into $S$. 
venezuelae ATCC15439. pSAPDK vector was integrated into the border near pikD through homologous recombination, and integration of pSAPDK was confirmed by PCR analysis (Additional file 1: Figure S1). HindIIIdigested total chromosomal DNA fractions were selfligated, after which they were transformed into E. coli cells. Transformed strains were selected using apramycin and kanamycin antibiotics and then confirmed by PCR, enzyme mapping, and sequencing (Additional file 2: Figure S2). Finally, the DNA fragment containing attPint $t_{\text {ФВT1 }}$ was re-introduced into the rescued recombinant pSBAC vector and named pPik001 (Fig. 1).

\section{Heterologous expression of pikromycin biosynthetic gene cluster in Streptomyces strains}

To confirm expression of the pikromycin biosynthetic gene cluster in pPik001, it was introduced into both $S$. lividans and $S$. coelicolor strains through conjugation, which generated S. lividans Pik101 and S. coelicolor Pik201, respectively. The constructed strains were cultured along with wild-type S. venezuelae ATCC 15439. Although pikromycin was not detected from wild-type or heterologous hosts, highly noticeable peaks showing identical retention times and spectra were detected in all three cultures (Fig. 3a, data not shown for S. coelicolor Pik201). The collected fraction of the peak was analyzed with high resolution LC/MS and confirmed to be 10-deoxymethynolide, a major pikromycin derivative released from module 5 in pikromycin biosynthetic gene cluster. The results of LC/MS also confirmed expression of the pikromycin biosynthetic gene cluster and that the yields of 10-deoxymethynolide in S. lividans Pik101 and S. coelicolor Pik201 were 346.8 and $396.2 \mathrm{mg} / \mathrm{L}$, respectively. When the production yield of the heterologous hosts was compared with the wild-type, S. lividans Pik101 and S. coelicolor Pik201 exhibited 1.6-fold and 1.8-fold increases, respectively (Fig. 2), revealing that the pSBAC-driven heterologous expression of an entire pikromycin biosynthetic gene cluster resulted in enhanced production in both S. lividans and S. coelicolor.

\section{Heterologous tandem integration of entire pikromycin cluster}

To further stimulate pikromycin productivity, an additional copy of the pikromycin cluster was introduced into S. lividans Pik101 containing a single-copy of pikromycin. To introduce the second copy of the pikromycin gene cluster, the apramycin resistance gene in pPik001 was substituted with a hygromycin resistance gene and named pPik003. Next, pPik003 was introduced into $S$. lividans Pik101 through conjugation, after which the

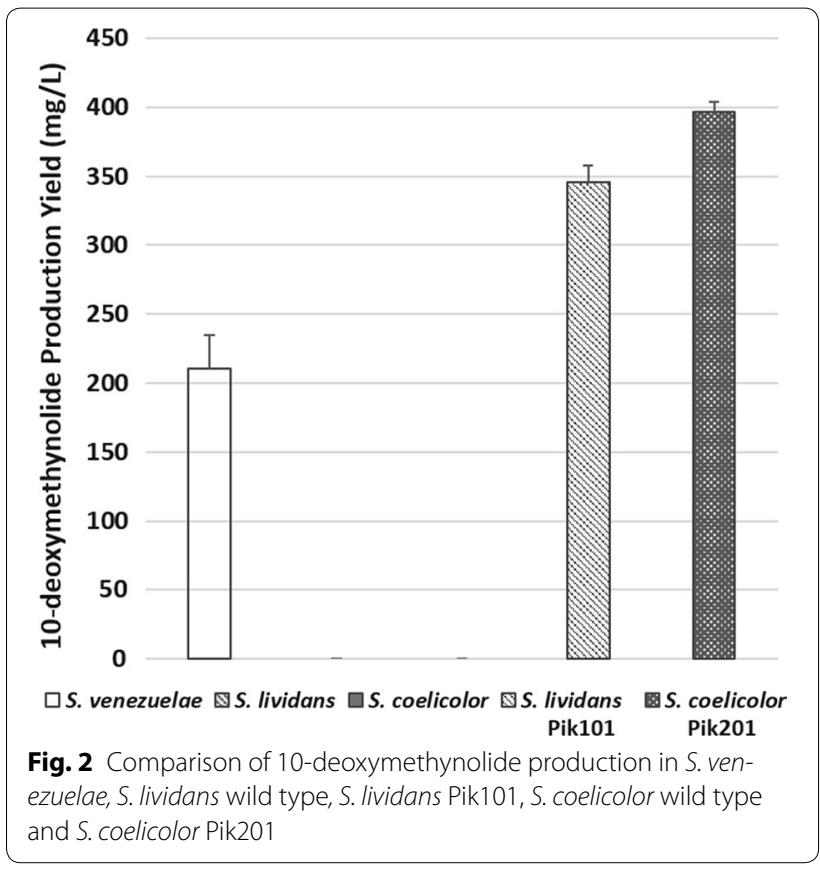

exconjugant was selected using both hygromycin and apramycin. Four exconjugants were randomly chosen to confirm the tandem integration by PCR amplification of attP-int primer sets. In all four selected colonies, the pPik003 was integrated into adjacent region of the pPik001 by homologous recombination. The resulting strain was named S. lividans Pik102. Although pikromycin was not detected in both S. venezuelae and S. lividans Pik101 as stated above, pikromycin production was observed in S. lividans Pik102 with the pikromycin yield of $333.7 \mathrm{mg} / \mathrm{L}$ (Fig. 3a). Moreover, the total production of pikromycin and 10-deoxymethynolide in S. lividans Pik102 (604.7 mg/L) showed a 2.1-fold increase compared to the production of only 10-deoxymethynolide in the parental wild-type strain $(280 \mathrm{mg} / \mathrm{L})$ (Fig. 3b). Additionally, the yield of S. lividans Pik102 was 1.2-fold higher than that of S. lividans Pik101. Production of the compounds in S. lividans Pik102 remained high until day 6 of culture, whereas production in S. lividans Pik101 gradually decreased from day 4 of culture (Fig. 3c). Comparative qRT-PCR results also confirmed that transcription of four biosynthetic genes (pikAI, pikC, desIII and desVI), as well as the pathway-specific regulatory gene pikD, was significantly stimulated in S. lividans Pik102 (Fig. 4). These findings imply that the presence of an additional copy of the entire pikromycin biosynthetic gene cluster was responsible for the increased transcription of pikromycin biosynthetic genes. 

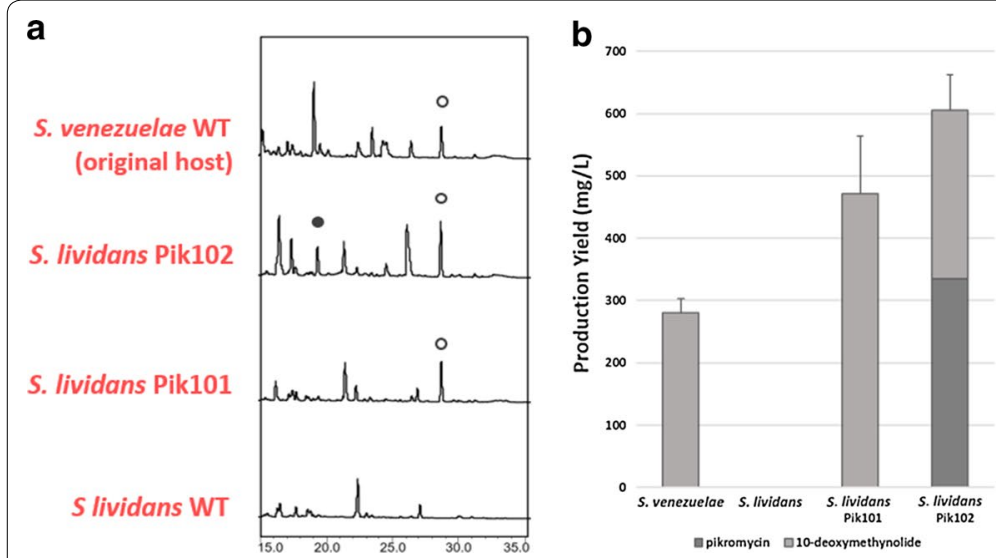

C

Fig. 3 a HPLC profiles of S, venezuelae, S. lividans Pik102, S. lividans Pik101 and S. lividans: open circle, 10-deoxymethynolide; closed circle, pikromycin b The production yield of pikromycin and 10-deoxymethynolide in S. venezuelae, S. lividans Pik101 and S. lividans Pik102. c The production yield of pikromycin and 10-deoxymethynolide in S. lividans Pik101 and S. lividans Pik102 for 4 days; open circle, S. lividans Pik101; closed circle, S. lividans Pik102

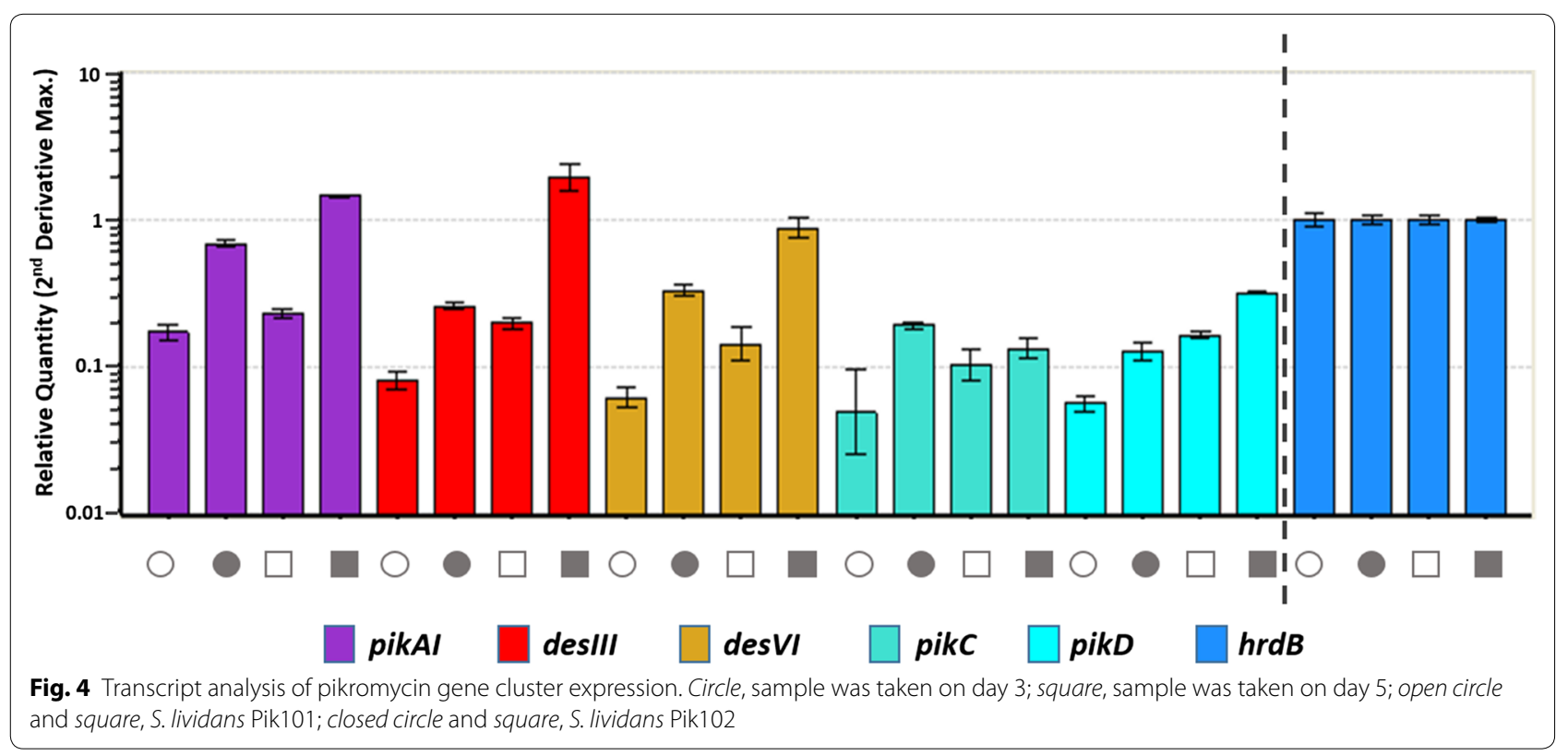

\section{Discussion}

The E. coli-Streptomyces shuttle vector, pSBAC, is an efficient tool that is capable of accepting and stably maintaining a large-sized natural product biosynthetic gene cluster [11]. Although we previously demonstrated an efficient strategy for giant plasmid rescue and tandem overexpression of a large-sized TMC biosynthetic gene cluster both in homologous and heterologous hosts [13], another example of a natural product biosynthetic gene cluster with different restriction enzyme site cloning was needed to be accepted as a general heterologous expression system in Streptomyces. Therefore, a pikromycin biosynthetic gene cluster was isolated using the pSBAC system as another example. The pikromycin biosynthetic gene cluster has some problems in that diverse derivatives were seen in a production culture, production varied in flasks and pikromycin production yield was quite low, making it difficult to detect. Because the pikromycin biosynthetic gene cluster was expressed in heterologous hosts, the production yield of 10-deoxymethynolide, one of pikromycin derivatives, was 1.6- and 1.8-fold of that of the parental wild-type host in S. lividans Pik101 and S. coelicolor Pik201, respectively. Moreover, pikromycin was detected in S. lividans Pik102, which has two copies of pikromycin biosynthetic gene cluster. These results suggest that a bottleneck exists in the pikromycin biosynthetic pathway that could be solved by introducing additional pikromycin biosynthetic genes. Expression of the pikromycin biosynthetic gene cluster 
was also confirmed through real-time RT-PCR at the transcriptional level. As expected, the level of transcripts of pikromycin biosynthetic genes in S. lividans Pik102 was higher than that in S. lividans Pik101 on both day 3 and 5. Although a significant gap in expression levels between S. lividans Pik101 and S. lividans Pik102 was shown, the compound yield in S. lividans Pik102 did not differ significantly from that of S. lividans Pik101. These findings suggest that the pikromycin gene cluster was amply transcribed, but that another bottleneck may exist in pikromycin production, such as a shortage of building blocks. In conclusion, the Streptomyces pSBAC-driven precise cloning and tandem integration approach described here can be applied to the versatile overexpression of large secondary metabolite gene clusters in actinomycetes, including novel cryptic clusters identified by genome mining.

\section{Conclusions}

Although actinomycetes have provided abundant sources for the development of new pharmaceuticals, their widespread application for this purpose is hindered by low productivity and difficult cultivation. Because many cryptic pathways have been found through genome mining, heterologous expression was suggested as a useful method for production of new pharmaceuticals. Development of an essential tool that can isolate, maintain, and express a large-sized gene cluster has emerged as a major point of interest. We previously demonstrated that the pSBAC system was an attractive genetic system for the efficient heterologous overexpression of a target cluster in Streptomyces species. However, the process for isolation of a biosynthetic gene cluster still requires a series of conjugation and selection steps. Here, we isolated the pikromycin biosynthetic gene cluster as a model pathway using the pSBAC system and simplified the process by eliminating one conjugation step using a HindIII restriction enzyme site in multiple cloning sites of pSBAC for plasmid rescue. Introduction of two copies of the pikromycin biosynthetic gene cluster into S. lividans was confirmed to stimulate the production of both 10-deoxymethynolide and pikromycin. Overall, the results of this study showed natural microbial products that are usually not produced or produced in very low amounts could be generated via overexpression of a biosynthetic gene cluster. This study confirms that the pSBAC system is a generally-applicable technology for the precise cloning and functional overexpression of the largesized biosynthetic gene cluster of any potentially-valuable low-titer metabolites in actinomycetes.

\section{Methods}

\section{Bacterial strains and culture media}

The bacterial strains and plasmids used in this study were summarized in Table 1. E. coli strains were cultured in
Luria-Bertani (LB) broth or on Luria-Bertani agar supplemented with appropriate antibiotics at $37{ }^{\circ} \mathrm{C}$. For production of pikromycin and its derivatives, the original host, S. venezuelae, was grown in SGGP media for 1 day, then cultured for 3 days in SCM media at $30{ }^{\circ} \mathrm{C}$ [15]. Additionally, the model strains S. lividans and S. coelicolor were grown in TSB media for 2 days, then cultured for 5 days in $\mathrm{R} 5$ media at $30^{\circ} \mathrm{C}$ [18]. Conjugation was carried out on modified ISP4 medium.

\section{Insertion of unique HindIII recognition sequence into a flanking region of the pikromycin biosynthetic gene cluster}

To isolate the pikromycin biosynthetic gene cluster, a unique HindIII recognition sequence was inserted into a flanking region of the pikromycin biosynthetic gene cluster using a PCR-targeted gene disruption system [17].

Table 1 Bacterial strains and plasmids used in this study

\begin{tabular}{|c|c|c|}
\hline Strain/plasmid & Relevant characteristics & $\begin{array}{l}\text { Source/ } \\
\text { references }\end{array}$ \\
\hline \multicolumn{3}{|l|}{ Plasmid } \\
\hline pSBAC & $\begin{array}{l}\text { aacIII(IV), oriT, attP-int } \\
\text { of pCC1BAC }\end{array}$ & {$[11]$} \\
\hline pSAPDK & $\begin{array}{l}\text { Modified pSBAC with deleted attP- } \\
\text { int }_{\Phi \mathrm{BT} 1} \text { and inserted } \mathrm{Kan}^{R} \text { and pikD } \\
\text { fragment }\end{array}$ & This work \\
\hline pPik & $\begin{array}{l}\text { PSAPDK with } 60 \text { kb DNA insert con- } \\
\text { taining whole pik gene cluster }\end{array}$ & This work \\
\hline pPik001 & pPik with attP-int $t_{\triangle \mathrm{BT} 1}$ & This work \\
\hline pPik003 & $\begin{array}{l}\text { pPik101, which replaced } \mathrm{Hyg}^{R} \text { with } \\
\mathrm{Apr}^{R}\end{array}$ & This work \\
\hline \multicolumn{3}{|l|}{ E. coli } \\
\hline EPI300 & $\begin{array}{l}\text { F-mcrA-D (mrr-hsdRMS-mcrBC) trfA } \\
\text { host for cloning and amplification of } \\
\text { various BAC vectors and constructs } \\
\text { derived from it }\end{array}$ & Epicenter \\
\hline S17-1 & $\begin{array}{l}\text { E. coli host for transferring various } \\
\text { plasmids into Streptomyces via } \\
\text { conjugation }\end{array}$ & \\
\hline $\begin{array}{l}\text { ET12567/ } \\
\text { pUZ8002 }\end{array}$ & $\begin{array}{l}\text { E. coli host for transferring various } \\
\text { plasmids into Streptomyces via } \\
\text { conjugation }\end{array}$ & \\
\hline \multicolumn{3}{|c|}{ Streptomyces venezuelae } \\
\hline ATCC 15439 & Original pikromycin-producing strain & {$[15]$} \\
\hline $\begin{array}{l}\text { 15439-Hindll- } \\
\text { IBAC }\end{array}$ & $\begin{array}{l}\text { ATCC } 15439 \text { with pSAPDK and HindIII } \\
\text { recognition sequences in a flanking } \\
\text { region of pikromycin biosynthetic } \\
\text { gene cluster }\end{array}$ & This work \\
\hline \multicolumn{3}{|c|}{ Streptomyces lividans } \\
\hline TK21 & Non pikromycin-producing strain & \\
\hline Pik101 & TK21 with pPik001 & This work \\
\hline Pik102 & Pik101 with pPik003 & This work \\
\hline \multicolumn{3}{|c|}{ Streptomyces coelicolor } \\
\hline M145 & Non pikromycin-producing strain & \\
\hline Pik201 & M145 with pPik001 & This work \\
\hline
\end{tabular}


Table 2 Primer list used in this study

\begin{tabular}{|c|c|c|}
\hline Oligonucleotide & Primer sequence $\left(5^{\prime}-3^{\prime}\right)$ & Purpose \\
\hline \multicolumn{3}{|c|}{ Homo arm-apr ${ }^{R}$ cassette } \\
\hline $\mathrm{F}$ & $\begin{array}{l}\text { TACCCGGCGGAGGCGTACCACCAGCAGTATCTGGACAAGAAAT } \\
\text { TCCGGGGATCCGTCGACC }\end{array}$ & Construction of pMSCPik311 \\
\hline R & $\begin{array}{l}\text { AGCGGCGCAGCCTCATGTAACGGGCCTGAGAGTGGCAGAAG } \\
\text { CTTGCATTGTAGGCTGGAGCTGCCTC }\end{array}$ & \\
\hline \multicolumn{3}{|l|}{ Apr $^{R}$ cassette check } \\
\hline $\mathrm{F}$ & TACGGCGAGATCACCACGAC & Check for insertion of $a p r^{R}$ cassette \\
\hline $\mathrm{R}$ & GCGAGGATCGAGACGCGTGA & \\
\hline \multicolumn{3}{|l|}{ PikD-kan } \\
\hline F & ACAACACCAAGCTTGCCCGCCT & Construction of pSAPDK \\
\hline $\mathrm{R}$ & ACTGCAAGCTAGGCCCACCGATGAAGGACA & \\
\hline $\mathrm{F}$ & TCGGTGGGCCTTAGCTTGCAGTGGGCTTACA & \\
\hline $\mathrm{R}$ & TTGGTCGGTGAATTCGAACCCCAGA & \\
\hline \multicolumn{3}{|l|}{ PikD-kan check } \\
\hline $\mathrm{F}$ & AGGCCCTGTTCGACGACCGA & Confirmation of pPik001 \\
\hline $\mathrm{R}$ & TGCCTCGTCCTGCAGTTCATTCA & \\
\hline \multicolumn{3}{|l|}{ PikRI-Al } \\
\hline $\mathrm{F}$ & CCCCTTCGAATTTCCGCCGC & Confirmation of pPik001 \\
\hline $\mathrm{R}$ & CCGCCAGGAGTTCCCAGAAC & \\
\hline \multicolumn{3}{|l|}{ PikAll } \\
\hline $\mathrm{F}$ & ACCGGTCTCAACTTCCGCGA & Confirmation of pPik001 \\
\hline $\mathrm{R}$ & TCCAGGAGGGACAGGGTCAGC & \\
\hline \multicolumn{3}{|l|}{ PikD } \\
\hline $\mathrm{F}$ & TCGCGGCACGGCATGGAGTACATGCA & Confirmation of pPik001 \\
\hline $\mathrm{R}$ & TCAGGCCGTGACGGACTTGTCCT & \\
\hline \multicolumn{3}{|l|}{ PikAI RNA } \\
\hline F & GACTGGGACCGCTTCTACCT & qRT-PCR \\
\hline $\mathrm{R}$ & TACGAGACCGAGGAGGATCT & \\
\hline \multicolumn{3}{|l|}{ DesIII RNA } \\
\hline F & GCTCTTTCTGGACCGCATAG & qRT-PCR \\
\hline $\mathrm{R}$ & CGGTCATTTCGAAGCAGATT & \\
\hline \multicolumn{3}{|l|}{ DesVI RNA } \\
\hline F & CATCTGGAGCACTTCACCAA & qRT-PCR \\
\hline $\mathrm{R}$ & CTCGGTCGTCTTCAGGTAGC & \\
\hline \multicolumn{3}{|l|}{ PikC RNA } \\
\hline $\mathrm{F}$ & GCTATCTCTCCCGGCTCATC & qRT-PCR \\
\hline $\mathrm{R}$ & GATTGACCGTGGTCTCGTG & \\
\hline \multicolumn{3}{|l|}{ PikD RNA } \\
\hline F & GGACGCTCTTACTCGTCTCC & qRT-PCR \\
\hline $\mathrm{R}$ & CGGAGTAACTGGCAGAGGAC & \\
\hline \multicolumn{3}{|l|}{ HrdB RNA } \\
\hline $\mathrm{F}$ & GCGGTGGAGAAGTTCGACTA & qRT-PCR \\
\hline $\mathrm{R}$ & CACATGGTCGAGGTCATCAA & \\
\hline
\end{tabular}

An apramycin resistance gene $(\operatorname{aac}(3) I V) / o r i T$ cassette was used to insert a HindIII recognition sequence into a flanking region near pikRII. This cassette was amplified from pIJ773 using the primers containing the Hin$d$ III recognition sequence and a homologous sequence with a flanking region near pikRII at the $5^{\prime}$ - and $3^{\prime}$-ends, respectively. The cassette was then introduced into $E$. coli BW25113/pIJ790 containing pSCPik311, resulting in pSCPik311::aac(3)IV/oriT, pMSCPik311. Insertion of the HindIII recognition sequence was confirmed by 
PCR amplification and sequencing (Macrogen, Korea). The mutated cosmid, pMSCPik311, was subsequently introduced into E. coli ET12567/pUZ8002 and then was directly conjugated with S. venezuelae ATCC 15439. Conjugation experiments were then performed as previously described [11].

\section{Isolation of entire pikromycin biosynthetic gene cluster into PSBAC}

To isolate the entire pikromycin biosynthetic gene cluster from the chromosome by HindIII digestion and ligation, attP-int $t_{\triangle \mathrm{BT} 1}$ was removed from pSBAC by AvrII digestion and self-ligation, and the modified vector was named pSA. To integrate pSA into the vicinity of pikD by homologous recombination and select the correct colonies, a cassette containing a 1879-bp DNA fragment including a portion of $p i k D$ and a kanamycin resistance gene was constructed by 3-way PCR amplification using primers containing the restriction enzyme sites, HindIII and EcoRI, respectively. The amplified PCR product was ligated into a T\&A cloning vector (RBC), after which the ligated vector was sequenced to confirm its integrity (Macrogen, Korea). The cassette was then digested using HindIII and EcoRI, after which it was cloned into EcoRIHindIII-digested pSA to generate pSAPDK. Conjugation was subsequently performed to integrate pSAPDK into the chromosomal DNA in S. venezuelae via homologous recombination. Next, the desired mutants were selected on apramycin- and kanamycin-containing ISP4 agar, verified using PCR and named S. venezuelae HindBAC. This strain was cultured in TSB media for 1 day at $30^{\circ} \mathrm{C}$, after which its genomic DNA was prepared using a Wizard ${ }^{\circledR}$ genomic DNA purification kit (Promega). Genomic DNA was then digested by restriction enzyme HindIII, purified, and concentrated by ethanol precipitation before self-ligation using T4 ligase (TaKaRa). After desalting, the ligation mixture was used for electroporation of $E$. coli EPI300. Recombinants were selected on apramycinand kanamycin-containing LB medium, after which plasmids were isolated by alkali denaturation and screened by PCR using PikRI-AI, PikAII, PikD and PikD-kan check primers in pikromycin cluster to identify pPik. The attP$i n t_{Ф \mathrm{BT} 1}$ was digested by $A v r \mathrm{II}$ from the attP-int $t_{\Phi_{\mathrm{BT}} 1^{-}}$ containing vector constructed in a previous study and then cloned into pPik to generate pPik001.

\section{Extraction and HPLC quantification of 10-deoxymethynolide}

Each $50 \mathrm{~mL}$ culture medium was centrifuged at $6000 \times g$ for $15 \mathrm{~min}$. Pikromycin and its derivatives were extracted from the supernatant with two volumes of ethyl acetate and concentrated using a rotary evaporator, after which the final extracts were dissolved in methanol. Analytical HPLC analysis was conducted using an Agilent C18 5 - $\mu \mathrm{m}$ column with $80 \%$ acetonitrile in $5 \mathrm{mM}$ ammonium acetate buffer containing $0.05 \%$ acetic acid at a flow rate of $1 \mathrm{~mL} / \mathrm{min}$. Detection was performed at $220 \mathrm{~nm}$ [19].

\section{Replacement of apramycin-resistant gene by other antibiotic resistance genes and introduction into pik-containing strain}

To introduce the additional pikromycin cluster into $S$. lividans Pik101, the apramycin-resistant gene of pPik001 was replaced by a hygromycin-resistant gene using a Quick and Easy BAC modification kit (GeneBridges), after which it was denoted pPik003. Next, pPik001 was introduced into Red/ET plasmid-containing E. coli EPI300, after which BAC modification was performed in accordance with the manufacturer's guide using the hygromycin-resistant gene containing the $a p r^{R}$-homologous arm constructed by PCR. Transformants were selected on hygromycin containing LB medium and confirmed by PCR. After pPik003 was transformed into $E$. coli S17-1, it was introduced into S. lividans Pik101 by conjugation.

\section{Isolation of total RNA and gene expression analysis by qRT-PCR}

For RNA preparation, S. lividans TK21, S. lividans Pik101 and S. lividans Pik102 were grown for 6 days in R5 medium, during which time samples were collected on day 3 and 5 . Mycelia were harvested by centrifugation and stored in a $-80{ }^{\circ} \mathrm{C}$ deep freezer after washing twice with distilled water. RNA preparation and qRT-PCR were then carried out as previously reported [20] using the primers shown in Table 2.

\section{Additional file}

Additional file 1: Figure S1. (A) Confirmation of Hindlll insertion near pikRI/ with $a p r^{R}$ check primers shown in Table 2. The expected amplicon size of mutant and wildtype is $1.5 \mathrm{~kb}$ and $360 \mathrm{bp}$, respectively; lane 1, 100 bp loading DNA ladder (DYNEBIO Inc.); lane 2 and 4, PCR products from S. venezuelae tDNA; lane 3 and 5, PCR products from S. venezuelae Hindbac tDNA; lane 4 and 5, Hindlll digested PCR products. (B) Confirmation of integration of PSAPDK in the vicinity of pikD; lane 1, $1 \mathrm{~kb}$ DNA ladder (cosmogenetech); lane 2, PCR product from S. venezuelae Hindbac tDNA; lane 3, PCR product from $S$. venezuelae tDNA.

Additional file 2: Figure S2. Confirmation of pPik001 (A) PCR analysis using randomly selected primer within pikromycin biosynthetic gene cluster. Amplicons were loaded on a gel in order of pikD-kan (lane 2-3), PikAll (lane 4-5), pikRI-pikAl (lane 6-7), pikD check primers (lane 8-9). Lane 1, $1 \mathrm{~kb}$ DNA ladder (Cosmo genetech); lane2, 4, 6, 8, S. venezuelae tDNA; lane 3,5,7,9, pPik001. (B) Enzyme mapping using various restriction enzymes. Digestion mixtures were loaded on a gel in order of $B g / l$ digestion (1-2), Pstl digestion (3-4), Bg/ll and Pstl digestion (5-6). M, $\lambda$-Hindlll DNA ladder; 1, 3, 5, pSBAC; 2, 4, 6, pPik001. 


\section{Authors' contributions}

HRP and HJN conducted the experiments, analyzed the primary data and drafted the manuscript. SHK and SSC participated in the data analysis. ESK supervised the research and revised the manuscript. All authors read and approved the final manuscript.

\section{Acknowledgements}

Not applicable.

\section{Competing interests}

The authors declare that they have no competing interests.

\section{Consent for publication}

The authors are consent for publication.

\section{Funding}

This work was conducted by the National Research Foundation of Korea (NRF) grant funded by the Korean government (MSIP) (No. NRF2014R1A2A1A11052236). This work was also supported with the support of the "Cooperative Research Program for Agriculture Science and Technology Development (Project No. PJ01129601)" Rural Development Administration, Republic of Korea.

\section{Publisher's Note}

Springer Nature remains neutral with regard to jurisdictional claims in published maps and institutional affiliations.

Received: 27 January 2017 Accepted: 18 May 2017

Published online: 31 May 2017

\section{References}

1. Donadio S, Monciardini P, Sosio M. Polyketide synthases and nonribosomal peptide synthetases: the emerging view from bacterial genomics. Nat Prod Rep. 2007;24(5):1073-109.

2. Galm U, Shen B. Expression of biosynthetic gene clusters in heterologous hosts for natural product production and combinatorial biosynthesis. Expert Opin Drug Discov. 2006;1(5):409-37.

3. Ylihonko K, Hakala J, Kunnari T, Mäntsälälä P. Production of hybrid anthracycline antibiotics by heterologous expression of Streptomyces nogalater nogalamycin biosynthesis genes. Microbiology. 1996;142(8):1965-72.

4. He J, Hertweck C. Iteration as programmed event during polyketide assembly; molecular analysis of the aureothin biosynthesis gene cluster Chem Biol. 2003:10(12):1225-32

5. Blodgett JA, Zhang JK, MetcalfW. Molecular cloning, sequence analysis, and heterologous expression of the phosphinothricin tripeptide biosynthetic gene cluster from Streptomyces viridochromogenes DSM 40736. Antimicrob Agents Chemother. 2005;49(1):230-40.

6. Gomez-Escribano JP, Bibb MJ. Engineering Streptomyces coelicolor for heterologous expression of secondary metabolite gene clusters. Microb Biotechnol. 2011:4(2):207-15.
7. Mantovani SM, Moore BS. Flavin-linked oxidase catalyzes pyrrolizine formation of dichloropyrrole-containing polyketide extender unit in chlorizidine A. J Am Chem Soc. 2013;135(48):18032-5.

8. Iftime D, Jasyk M, Kulik A, Imhoff JF, Stegmann E, Wohlleben W, Süssmuth RD, Weber T. Streptocollin, a type IV lanthipeptide produced by Streptomyces collinus Tü 365. ChemBioChem. 2015;16(18):2615-23.

9. Fu J, Bian X, Hu S, Wang H, Huang F, Seibert PM, Plaza A, Xia L, Müller R, Stewart AF, Zhang Y. Full-length RecE enhances linear-linear homologous recombination and facilitates direct cloning for bioprospecting. Nat Biotechnol. 2012;30(5):440-6.

10. Yamanaka K, Reynolds KA, Kersten RD, Ryan KS, Gonzalez DJ, Nizet V, Dorrestein PC, Moore BS. Direct cloning and refactoring of a silent lipopeptide biosynthetic gene cluster yields the antibiotic taromycin A. Proc Natl Acad Sci USA. 2014;111(5):1957-62.

11. Liu H, Jiang H, Haltli B, Kulowski K, Muszynska E, Feng X, Summers M, Young M, Graziani E, Koehn F, Carter GT, He M. Rapid cloning and heterologous expression of the meridamycin biosynthetic gene cluster using a versatile Escherichia coli-Streptomyces artificial chromosome vector, pSBAC. J Nat Prod. 2009;72(3):389-95.

12. Yin J, Hoffmann M, Bian X, Tu Q, Yan F, Xia L, Ding X, Stewart AF, Muller R, Fu J, Zhang Y. Direct cloning and heterologous expression of the salinomycin biosynthetic gene cluster from Streptomyces albus DSM41398 in Streptomyces coelicolor A3(2). Sci Rep. 2015;5:15081.

13. Nah H, Woo M, Choi S, Kim E. Precise cloning and tandem integration of large polyketide biosynthetic gene cluster using Streptomyces artificial chromosome system. Microb Cell Fact. 2015;14(1):1.

14. Brockmann $\mathrm{H}$, Henkel W. Pikromycin, ein bitter schmeckendes Antibioticum aus Actinomyceten (Antibiotica aus Actinomyceten, VI. Mitteil. Chem Ber. 1951:84(3):284-8.

15. Xue Y, Sherman DH. A gene cluster for macrolide antibiotic biosynthesis in Streptomyces venezuelae: architecture of metabolic diversity. Proc Natl Acad Sci USA. 1998;95(21):12111-6.

16. Dutta S, Whicher JR, Hansen DA, Hale WA, Chemler JA, Congdon GR, Narayan AR, Håkansson K, Sherman DH, Smith JL, Skiniotis G. Structure of a modular polyketide synthase. Nature. 2014;510(7506):512-7.

17. Gust B, Challis GL, Fowler K, Kieser T, Chater KF. PCR-targeted Streptomyces gene replacement identifies a protein domain needed for biosynthesis of the sesquiterpene soil odor geosmin. Proc Natl Acad Sci USA 2003;100(4):1541-6.

18. Choi SS, Hur YA, Sherman DH, Kim ES. Isolation of the biosynthetic gene cluster for tautomycetin, a linear polyketide T cell-specific immunomodulator from Streptomyces sp. CK4412. Microbiology. 2007:153(4):1095-102.

19. Woo M, Nah H, Choi S, Kim E. Pikromycin production stimulation through antibiotic down-regulatory gene disruption in Streptomyces venezuelae. Biotechnol Bioprocess Eng. 2014;19(6):973-7.

20. Nah J, Park S, Yoon H, Choi S, Lee C, Kim E. Identification and characterization of wblA-dependent tmcT regulation during tautomycetin biosynthesis in Streptomyces sp. CK4412. Biotechnol Adv. 2012;30(1):202-9.

\section{Submit your next manuscript to BioMed Central and we will help you at every step:}

- We accept pre-submission inquiries

- Our selector tool helps you to find the most relevant journal

- We provide round the clock customer support

- Convenient online submission

- Thorough peer review

- Inclusion in PubMed and all major indexing services

- Maximum visibility for your research

Submit your manuscript at www.biomedcentral com/submit
Ciomed Central 